\title{
Gestión de la logística humanitaria en las etapas previas al desastre: revisión sistemática de la literatura
}

\section{Humanitarian logistics management in the previus stages to the disaster: systematic review of the literature}

\author{
Juan Camilo López-Vargas' ${ }^{1}$ \\ Diana María Cárdenas-Aguirre ${ }^{2}$
}

Recibido: mayo 31 de 2016 Aceptado: agosto 31 de 2016

\begin{abstract}
The aim of this paper is to analyze how mitigation and preparedness stages that are part of the process of humanitarian logistics are presented in the literature related with the topic. For this, a systematic review of the literature was conducted using application Tree of Science of the Universidad Nacional de Colombia in Manizales. The review allows recognize the importance that these stages have on the level of disaster response, as well as the types of disasters and mitigation and preparation decisions most commonly addressed. Likewise, a smaller participation of these topics is evidenced in the specialized literature. Therefore, extend the study of the stages of mitigation and preparedness to other types of disasters and other geographical contexts is a field of interest for future research.
\end{abstract}

Keywords: humanitarian logistics, Tree of Science, disaster mitigation, disaster preparedness.

Palabras clave: logística humanitaria, Tree of Science, mitigación de desastres, preparación de desastres. 


\section{Introducción}

La gestión logística de las operaciones de atención de desastres, llamada también logística humanitaria (Baldini, et al., 2012), es una disciplina que se ha desarrollado en respuesta a la creciente severidad y frecuencia de los desastres, tanto naturales como causados por el hombre (Camacho-Vallejo, et al., 2015). Los efectos de los desastres son devastadores en pérdidas humanas y materiales (Barbarosoğlu \& Arda, 2004; Oloruntoba \& Gray, 2009; Kunz, et al., 2014), y ponen a prueba la capacidad de las naciones para proteger de manera efectiva su infraestructura y población y recuperarse rápidamente (Altay \& Green, 2006).

La logística humanitaria se encarga de estimar, proveer, almacenar, transportar y distribuir el personal y servicios requeridos en la zona afectada por un desastre (Sharif \& Salari, 2015), a través de una gran diversidad de actividades desarrolladas en distintos momentos, que tienen como fin asistir a las personas sobrevivientes de un desastre (Kovács \& Spens, 2007). El objetivo principal es proveer suministros de emergencia rápidamente a las áreas afectadas, minimizando las muertes y el sufrimiento humano (Balcik \& Beamon, 2008).

Según Haghani y Oh (1996), el principal problema logístico es llevar un número de diferentes productos a través de distintos medios de transporte desde unos puntos de origen a uno o más destinos de forma efectiva y eficiente. Las decisiones respecto al qué comprar, en qué lugar, en qué momento, quién hace la compra, y de qué forma, resultan críticas en este tipo de contextos y conllevan serias implicaciones logísticas (Matopoulus, et al., 2014).

La logística humanitaria involucra todas las acciones dirigidas a la mitigación del riesgo, a reducir el impacto del desastre y a retornar al estado de normalidad (Hoyos, et al., 2015), las cuales se dividen en cuatro etapas: mitigación, preparación, respuesta y recuperación (Van Wassenhove, 2006; FEMA, 2010; Afsar, et al., 2014; Anaya-Arenas, et al., 2014; Rivera, et al., 2015). Este enfoque de cuatro etapas permite abarcar la planeación del sistema desde los niveles estratégico, táctico y operativo (Rancourt, et al., 2015).
Las actividades propias de las etapas de mitigación y preparación se desarrollan previo a la ocurrencia del desastre. La etapa de mitigación incluye el estudio de riesgos y los análisis estructurales (Yi \& Özdamar, 2007), así como la determinación de los posibles usos del suelo y la infraestructura, para asegurar la accesibilidad a estas zonas (Kovács \& Spens, 2009). El reforzamiento de las edificaciones y las estructuras viales también hace parte de esta etapa y son de suma importancia respecto a la eficiencia que tendrá la respuesta al desastre (İvgin, 2013). La etapa de preparación incluye todas las actividades desarrolladas por la población, los gobiernos locales y otras organizaciones antes de que acontezca el desastre con el fin de reducir sus efectos potenciales (Kunz, et al., 2014). Algunas actividades de preparación son: el reclutamiento de voluntarios, el entrenamiento de la comunidad en la zona vulnerable, la adquisición de vehículos y equipos de emergencia, la compra y almacenamiento de suministros de emergencia, y el diseño de los planes de emergencia (Altay \& Green, 2006).

Por su parte, las etapas de respuesta y recuperación se adelantan posteriormente a la ocurrencia del desastre. Así, la fase de respuesta inicia inmediatamente acontece el desastre, con actividades como: búsqueda y rescate de personas, recolección de información sobre el área del desastre, identificación de los sitios apropiados para los refugios, determinación de rutas de evacuación, transporte y entrega de suministros de emergencia (Tzeng, et al., 2007; Holguín-Veras, et al., 2013). El objetivo es la preservación de la vida, las instalaciones, el ambiente, y la estructura social, económica y política de la comunidad, mientras se busca tener eficiencia en las operaciones (Altay \& Green, 2006; Lassiter, et al., 2015). Según Rottkemper, et al. (2011), la transición entre las etapas de respuesta y recuperación se hace de forma gradual. La recuperación incluye las acciones ejecutadas en el largo plazo que permiten a la comunidad estabilizarse y retornar a su situación de normalidad (Altay \& Green, 2006).

El proceso de la logística humanitaria presenta un comportamiento cíclico, ya que las situaciones vividas en la respuesta al desastre y las lecciones aprendidas en la fase de recuperación, pueden verse reflejadas en obras de reconstrucción y nue- 
vos planes de mitigación que reduzcan los riesgos de nuevos desastres (Baldini, et al., 2012; Hadiguna, et al., 2014; Scholten, et al., 2014; Hoyos, et al., 2015).

A pesar de la importancia que tiene cada etapa en el proceso de la logística humanitaria, Rodríguez-Espíndola y Gaytán (2015) afirman que las fases previas al desastre continúan siendo poco estudiadas por la investigación de operaciones y las ciencias de la administración, y han recibido poca atención en la literatura especializada. Con lo anterior, se evidencia la necesidad de aumentar los estudios relacionados con el tema, justificando así la intención de los autores por llevar a cabo una revisión sistemática de literatura que se oriente hacia las fases de mitigación y preparación en el marco de la logística humanitaria. A partir de los elementos considerados en revisiones anteriores, se ha elaborado la tabla 1 con el fin de mostrar los criterios tomados por otros autores y se compara con los elementos que fueron incluidos en el proceso de revisión para el presente trabajo.

\begin{tabular}{|c|c|c|c|c|c|c|c|c|}
\hline Criterios & $\begin{array}{l}\text { Abidi, et } \\
\text { al. (2014) }\end{array}$ & $\begin{array}{l}\text { Altay y } \\
\text { Green } \\
(2006)\end{array}$ & $\begin{array}{l}\text { Anaya- } \\
\text { Arenas, et } \\
\text { al. (2014) }\end{array}$ & $\begin{array}{l}\text { Hoyos, et } \\
\text { al. (2015) }\end{array}$ & $\begin{array}{c}\text { Özdamar } \\
\text { y Ertem } \\
\text { (2015) }\end{array}$ & $\begin{array}{l}\text { Pazirandeh } \\
\text { (2011) }\end{array}$ & $\begin{array}{c}\text { Pettit y } \\
\text { Beresford } \\
\text { (2009) }\end{array}$ & $\begin{array}{c}\text { Este } \\
\text { artículo }\end{array}$ \\
\hline $\begin{array}{l}\text { Descripción de } \\
\text { fuentes }\end{array}$ & $\mathrm{x}$ & $x$ & $\mathrm{x}$ & $\mathrm{x}$ & & $\mathrm{x}$ & & $\mathrm{x}$ \\
\hline $\begin{array}{l}\text { Nacionalidad de } \\
\text { los autores }\end{array}$ & $x$ & $x$ & & & & & & \\
\hline $\begin{array}{l}\text { Año de } \\
\text { publicación }\end{array}$ & $x$ & $x$ & & $x$ & & $x$ & & \\
\hline $\begin{array}{l}\text { Enfoque } \\
\text { investigativo }\end{array}$ & & & & & & & & $x$ \\
\hline $\begin{array}{l}\text { Metodología } \\
\text { utilizada }\end{array}$ & $x$ & $\mathrm{x}$ & & $x$ & $\mathrm{x}$ & $x$ & & \\
\hline $\begin{array}{l}\text { Etapas de } \\
\text { la logística } \\
\text { humanitaria }\end{array}$ & $x$ & $\mathrm{x}$ & & $\mathrm{x}$ & & & & $x$ \\
\hline $\begin{array}{l}\text { Funciones } \\
\text { logísticas }\end{array}$ & & & & & $x$ & $x$ & & $x$ \\
\hline Tipo de desastre & & $x$ & & & & & & $\mathrm{x}$ \\
\hline $\begin{array}{l}\text { Tipo de } \\
\text { contribución }\end{array}$ & $\mathrm{x}$ & $x$ & & & & & & \\
\hline $\begin{array}{l}\text { Factores críticos } \\
\text { de éxito }\end{array}$ & & & & & & & $x$ & \\
\hline $\begin{array}{l}\text { Indicadores de } \\
\text { rendimiento }\end{array}$ & $x$ & & & & & & & \\
\hline $\begin{array}{l}\text { Problema de } \\
\text { investigación }\end{array}$ & & & $x$ & $x$ & $x$ & & & \\
\hline Función objetivo & & & & $x$ & $x$ & $x$ & & \\
\hline $\begin{array}{l}\text { Parámetros } \\
\text { estocásticos }\end{array}$ & & & & $x$ & & & & \\
\hline $\begin{array}{l}\text { Sistemas de } \\
\text { información }\end{array}$ & & & & & $\mathrm{x}$ & & & \\
\hline $\begin{array}{l}\text { Alcance de } \\
\text { estudio }\end{array}$ & & & & & & & & $x$ \\
\hline Énfasis del estudio & & & & & & & & $x$ \\
\hline Zona geográfica & & & & & & & & $\mathrm{X}$ \\
\hline
\end{tabular}

Tabla 1. Criterios utilizados por los autores en anteriores revisiones de literatura. 
Como se ve en la tabla 1, ya se han hecho esfuerzos por definir las tendencias investigativas en la literatura especializada relacionada con la logística humanitaria, por lo que este trabajo se ha diseñado con el interés particular de presentar elementos novedosos, que no han incluido otros autores en sus respectivas revisiones. Un aspecto importante frente a otros artículos de revisión es el periodo de revisión, pues se tuvieron en cuenta artículos desde el año 1996 hasta el 2016. De igual forma se tuvieron en cuenta otros criterios, como el enfoque investigativo de los autores consultados, los tipos específicos de los desastres tratados en la literatura, la zona geográfica de los casos estudiados, el alcance y énfasis de los estudios, el análisis concreto en las etapas de mitigación y preparación de desastres, así como la herramienta del Tree of Science utilizada en el proceso de revisión de literatura. Igualmente, este trabajo destina un apartado importante para presentar resultados de carácter conceptual, enfocado en las etapas previas del desastre.

El proceso de revisión de literatura se llevó a cabo con el objetivo de identificar la forma en que los autores referenciados han abordado las etapas de mitigación y preparación de la logística humanitaria, identificando tendencias en los procesos de investigación, las características de los casos tratados en la literatura y los campos futuros de investigación alrededor de la temática.

El artículo se ha estructurado de esta manera: la siguiente sección describe la metodología utilizada en el proceso de revisión de la literatura. Posteriormente, se presentan los principales hallazgos obtenidos a partir de la revisión, mediante un análisis de los enfoques y alcances de los modelos propuestos en la literatura aplicados en las etapas de mitigación y preparación, con base en los elementos incluidos en la tabla 1. La sección final está dedicada a presentar las conclusiones del proceso de revisión realizado y las líneas de investigación futuras.

\section{Metodología}

El proceso de revisión de literatura comenzó con una búsqueda inicial en el sitio Web of Science. Esta búsqueda se realizó en las bases de datos de la "Colección principal de Web of Science", utilizando la ecuación de búsqueda "humanitarian" AND "logistics". Los criterios de búsqueda se aplicaron por "Tema" y como periodo de tiempo se seleccionó la opción "Todos los años". Estos elementos fueron aplicados el día 5 de febrero de 2016 y la búsqueda arrojó un total de 229 artículos.

Sobre los 229 artículos se realizó un refinamiento con la aplicación web del Tree of Science, ToS, de la Universidad Nacional de Colombia, sede Manizales. El ToS toma la información de cada artículo para construir una red de citaciones bibliográficas y a partir del número de veces que los artículos son citados, toma los 80 artículos de mayor participación en la red para clasificarlos como raíz, tronco y hojas. La analogía de un árbol en el ToS, establece que los artículos ubicados en la raíz corresponden a autores muy citados y se entienden como la base y sustento de la teoría, mientras que aquellos asignados al tronco corresponden a autores que citan a otros pero que también son citados, es decir, son los que dan estructura al tema de investigación. Por último, en las hojas se ubican los autores que citan mucho pero aún no son muy citados, se comprenden como las perspectivas y avances actuales del tema de estudio (Robledo, et al., 2014).

A partir del proceso descrito se obtuvo un grupo final de 80 artículos, a través del cual se desarrolló la revisión de literatura. La revisión de los artículos se orientó, en primer lugar, a identificar las características, naturaleza e importancia de las etapas de mitigación y preparación en el contexto de la logística humanitaria. Posteriormente, se tomaron los autores que concretamente dirigieron sus estudios en alguna de las etapas de mitigación y preparación, identificando el enfoque de investigación utilizado, sea cualitativo o cuantitativo; en los trabajos cuantitativos se identificó: (i) el tipo de desastre tratado, (ii) la zona geográfica donde se 
aplicó el estudio, (iii) el alcance planteado, es decir, si el estudio se definió para casos generales o para casos específicos, y (iv) el énfasis del estudio, esto es, si se trataron casos previos o se plantea- ban eventos futuros. La figura 1 esquematiza la metodología descrita en el proceso de revisión de literatura.

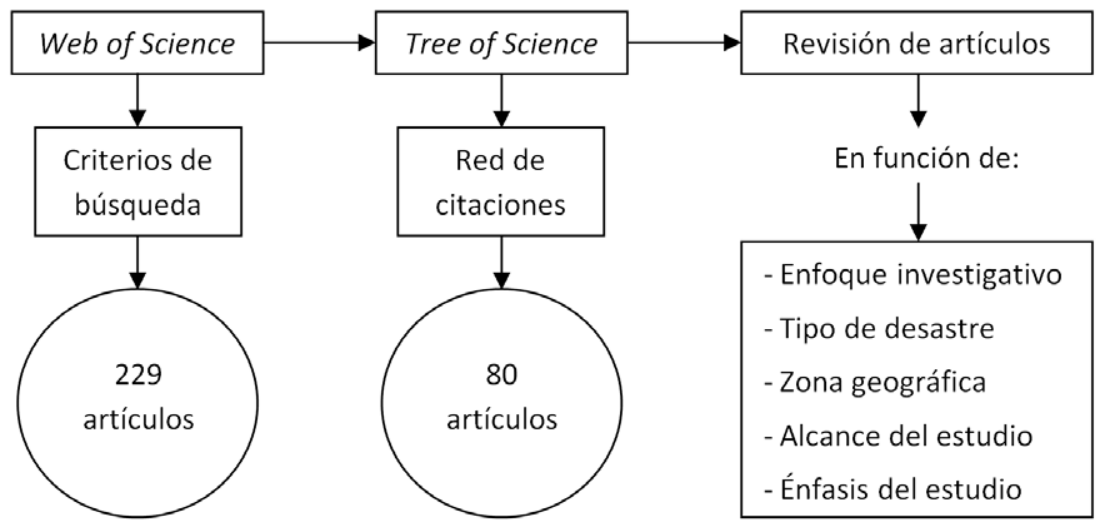

Figura 1. Proceso de revisión de la literatura con la aplicación ToS.

El análisis de los criterios definidos en la revisión tiene como propósito reconocer la forma en que las etapas de mitigación y preparación son abordadas desde la literatura especializada. Los resultados de la revisión de la literatura son presentados en la siguiente sección.

\section{Análisis de resultados}

Los resultados de esta revisión se clasifican en dos tipos, unos de naturaleza conceptual, que buscan caracterizar las etapas de mitigación y preparación, a través de la identificación de las funciones que cumplen, las decisiones que se toman y las dificultades que enfrentan, y una segunda, resultante del análisis de los criterios seleccionados para la revisión.

\subsection{Resultados de tipo conceptual}

Un primer momento de la revisión se dirigió a encontrar elementos que validaran la presencia e importancia que las etapas de mitigación y preparación tienen dentro de la logística humanitaria, y en el proceso de atención de desastres. Es así como, durante la lectura de los documentos selecciona- dos, se encontraron las siguientes posiciones sobre el tema.

Sobre la relevancia de las etapas de mitigación y preparación en el proceso de la logística humanitaria:

La literatura consultada en esta revisión resalta la importancia de las etapas previas a la ocurrencia y atención del desastre, proponiéndolas como factores clave de éxito en el logro de los objetivos de minimización de los impactos y mejoramiento de la eficiencia y efectividad en la respuesta. De esta forma, Tzeng et al. (2007), argumentan que el método más efectivo para reducir el impacto de un desastre es a través de la prevención, el monitoreo, la comunicación y la educación.

Para Ozguven y Ozbay (2015) las etapas previas al desastre son las más importantes de los procesos logísticos y deben comenzar estableciendo las relaciones necesarias entre los gobiernos, las fuerzas militares, las agencias civiles y el sector privado. Por lo tanto, una mejor preparación debe llevar a una fase de respuesta mucho más eficiente, siempre que la logística sea entendida como un elemento intrínseco en las operaciones de emergen- 
cia; es decir, se debe considerar la logística como un puente entre la preparación y la respuesta al desastre (Van Wassenhove, 2006; Kovács \& Spens, 2007).

Para Day et al. (2012), es a través del diseño, la implementación y la gestión de una cadena logística humanitaria que se pueden reducir los costos operacionales, las pérdidas de vidas y el sufrimiento de la comunidad afectada.

Los elementos aportados hasta aquí muestran la importancia de tomar anticipadamente las decisiones logísticas, desarrollar programas de prevención de desastres y adelantar planes de acción de respuesta, para llevar a cabo las operaciones de atención de desastres de manera rápida y eficiente, buscando reducir el impacto y el número de víctimas mortales.

Sobre las funciones y decisiones propias de las etapas de mitigación y preparación en el proceso de la logística humanitaria:

La literatura muestra que las etapas de mitigación y preparación cumplen, en el proceso de logística humanitaria, el propósito de diseñar la cadena de atención, mediante acciones como identificar y preparar a los actores, seleccionar las ubicaciones para los centros de acopio o trazar las rutas de distribución de ayudas. Los siguientes apartes de los artículos revisados son evidencia de ello:

Verma y Gaukler (2015), sostienen que la ubicación de los sitios para el almacenamiento de los suministros de emergencia es un factor crítico para la calidad del servicio prestado luego del desastre.

Kunz, et al. (2014) argumentan que, dirigiendo los esfuerzos en actividades como el reclutamiento y entrenamiento de personal, la educación de las comunidades, el aprendizaje de experiencias previas y el desarrollo de mejores prácticas, se pueden reducir sustancialmente los costos de preparación compensando las restricciones de presupuesto.
Señalan, además, que los planes de preparación bien desarrollados juegan un papel crucial en el rendimiento y efectividad de la respuesta al desastre.

El éxito de la logística humanitaria depende de la ejecución temprana de las actividades logísticas, la coordinación de los actores, la prontitud de la respuesta y la eficiente organización y entrega de los suministros y servicios de emergencia (Ülkü, et al., 2015). De esta manera, se pueden evitar inconvenientes e ineficiencias relacionadas con la falta de atención a la población.

Sobre las dificultades y retos que se enfrentan desde las etapas de mitigación y preparación en el proceso de la logística humanitaria

Teniendo en cuenta que las actividades de mitigación y preparación se desarrollan de manera previa a la ocurrencia del desastre, razón por la cual están exentas de la presión de la urgencia, resulta fundamental que quienes están a cargo de su desarrollo anticipen y preparen alternativas para las contingencias y dificultades propias de las etapas de respuesta y recuperación. Los artículos revisados señalan algunas de estas preocupaciones, así:

Según Maya-Duque, et al. (2016), la imposibilidad de entregar suministros en las zonas y a la gente que los necesita, es un factor que incide más en el incremento de las víctimas que la falta misma de suministros. Esta idea es complementada por Kovács y Spens, (2007), para quienes los principales problemas logísticos están relacionados con la falta de coordinación en la entrega de suministros, la demanda impredecible y el problema de distribución de la última milla.

Para Balcik y Beamon, (2008), la implementación de los planes de emergencia presenta serias restricciones debido a que la ubicación de instalaciones y el almacenamiento de equipos y suministros pueden resultar muy costosos, y esto puede llevar a proyectos financieramente inviables. 
La exploración de estas necesidades, sumada a las funciones y decisiones a cumplir en estas etapas del proceso, permite una aproximación a las áreas a las cuales debe dirigirse la investigación en el futuro.

3.2Resultados relacionados con los criterios utilizados para la revisión

Con el fin de caracterizar la actividad investigativa sobre el tema, se aplicaron tres criterios de análisis a los artículos revisados: el tipo de estudio adelantado, la etapa del proceso logístico a la cual va dirigido el estudio y la naturaleza del desastre que se estudia. Los resultados en cada una de estas dimensiones, se presentan a continuación.

\section{Respecto al enfoque de investigación que se utiliza}

Como se mencionó anteriormente, la revisión se adelantó sobre una base de 80 artículos, de los cuales se encontraron 26 trabajos de carácter cualitativo, entre los que se incluyen revisiones de literatura, modelos conceptuales y modelos de gestión para el diseño estratégico de las cadenas de suministro en el contexto de la logística humani- taria. Por otro lado, 49 de los 80 artículos revisados proponen y desarrollan modelos cuantitativos, es decir, el $61 \%$ de las publicaciones se enfocan en el desarrollo de modelos matemáticos para la toma de decisiones y en el modelamiento para simular o programar operaciones logísticas en el marco de la atención de desastres. Lo anterior concuerda con Hoyos, et al. (2015), para quienes la implementación de herramientas y técnicas de modelamiento debe ser un factor relevante tanto para las etapas de preparación como para las etapas posteriores al desastre. Es necesario comentar en este punto, que 5 artículos no fueron tenidos en cuenta ya que su enfoque no estaba relacionado con la logística humanitaria y por tanto no concordaban con el objetivo de la revisión.

Respecto a la etapa del proceso logístico que se considera en el estudio

Entre los 49 artículos que siguen un enfoque cuantitativo, un alto porcentaje se dedica a modelar las actividades de solo una o dos etapas del proceso logístico humanitario. La figura 2 muestra los artículos clasificados según las etapas tratadas por los autores consultados.

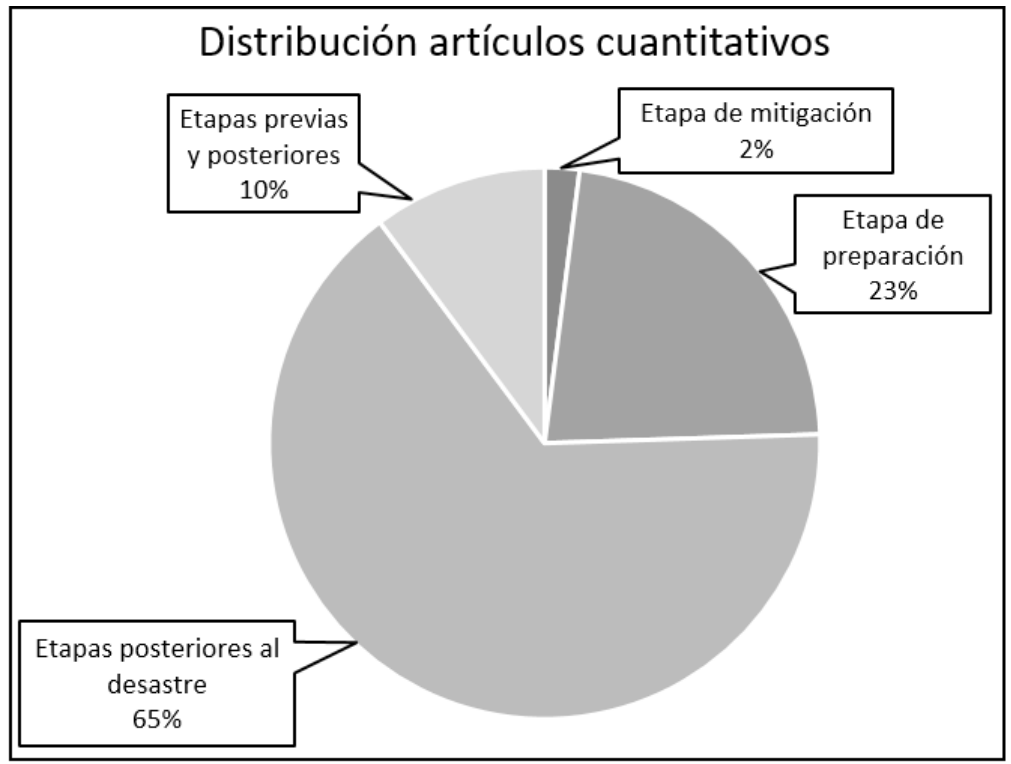

Figura 2. Distribución de los artículos cuantitativos en función de las etapas de la logística humanitaria. 
Teniendo en cuenta que el énfasis de este trabajo son las etapas previas al desastre, los artículos que trataban etapas posteriores al desastre se agruparon de forma indistinta en la figura 2 , sea que estudiaran la etapa de respuesta, recuperación, o ambas. Se tiene que el $10 \%$ de los artículos revisados, es decir, solamente 5 publicaciones, proponen modelos que comprenden al menos una de las etapas previas al desastre y al menos una de las etapas posteriores al desastre. Mientras que 11 artículos (el $23 \%$ de las publicaciones) se dedican específicamente a operaciones relacionadas con la etapa de preparación. Llama la atención que solo 1 artículo (el $2 \%$ que se observa en la figura 2) proponga un modelo heurístico aplicable a la etapa de mitigación. Con el fin de presentar las funciones logísticas planteadas en los modelos matemáticos encontrados en la literatura, se ha elaborado la tabla 2 para mostrar las propuestas realizadas por los autores en las etapas de mitigación y preparación.

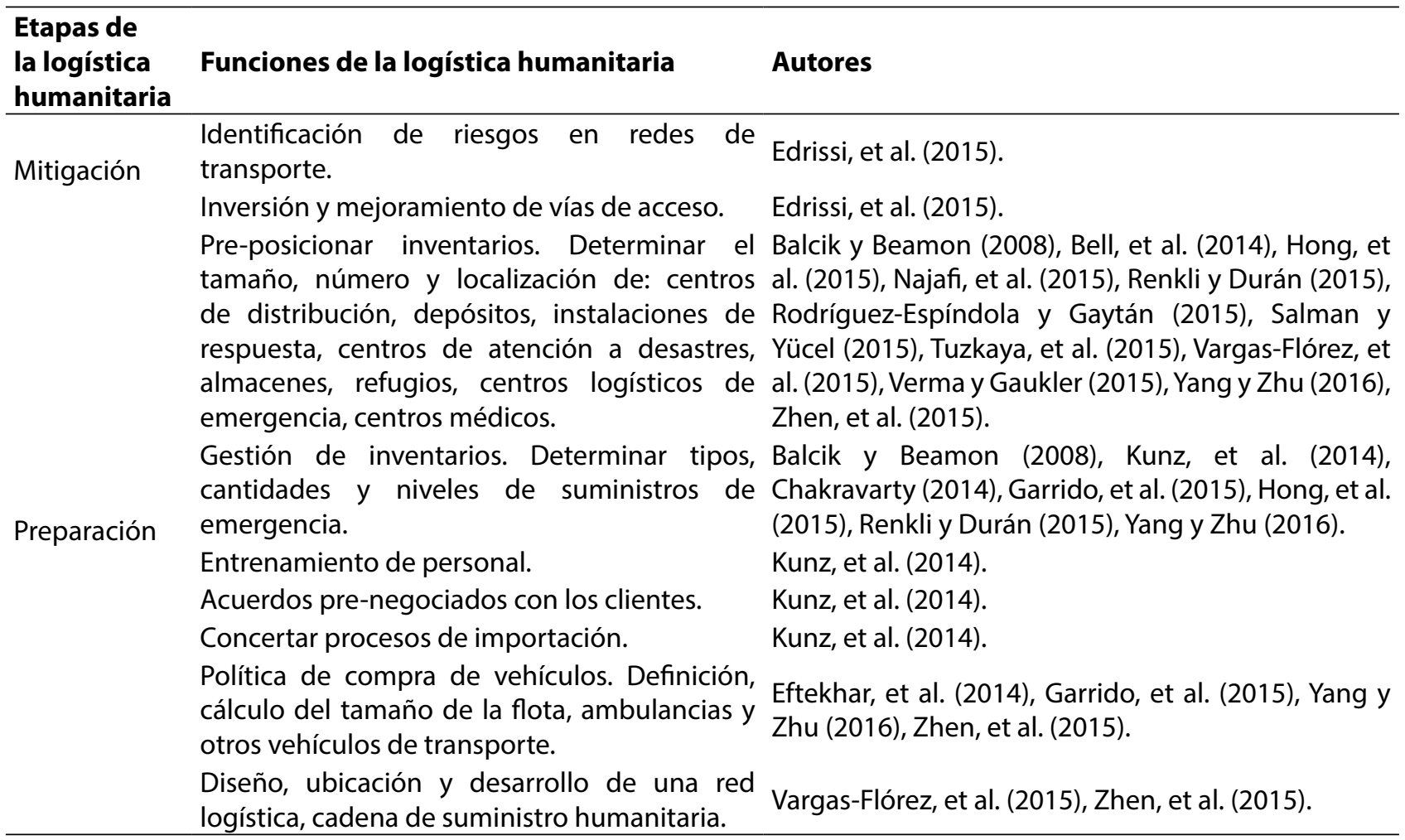

Tabla 2. Funciones logísticas en las etapas de mitigación y preparación.

Como se observa en la tabla 2, hay una tendencia hacia los problemas de localización de instalaciones y de definición de niveles de inventarios, previo a la ocurrencia del desastre. De acuerdo a Gutjahr y Dzubur, (2016), resulta evidente el mayor interés en la localización de centros de distribución y otras instalaciones, donde los afectados son abastecidos de productos o servicios de distinto tipo. El trabajo de Edrissi, et al. (2015), se enfoca en la etapa de mitigación aplicando su modelo hacia el estudio de riesgos en las vías cercanas a las zonas vulnerables. Kunz, et al. (2014) se centran en otras actividades de preparación, como el entrenamiento de personal, los pre-acuerdos con los proveedores y procesos de importación. Los modelos de Vargas-Flórez, et al. (2015) y Zhen, et al. (2015) mezclan objetivos de localización, inventario y definición de rutas de transporte, dirigidos 
al diseño de una red logística o modelo de aprovisionamiento humanitario.

Debido a la incertidumbre que caracteriza la logística humanitaria, los modelos matemáticos propuestos para la fase de preparación son generalmente estocásticos, usando escenarios probabilísticos para representar los desastres y sus consecuencias (Liu \& Guo, 2014; Özdamar y Ertem, 2015). Das y Hanaoka (2014), señalan que la mayoría de los autores se concentran en los aspectos de la logística comercial, los cuales tienen poca aplicabilidad en la logística humanitaria dadas las considerables diferencias en sus respectivas dinámicas.
Respecto al tipo de desastre que se estudia

Un análisis complementario de los modelos propuestos por los autores de la tabla 2, consideró las siguientes características: (i) si los estudios trataban casos específicos o generales, (ii) en dónde se aplicaron los modelos para los casos específicos, (iii) si los autores analizan eventos ya ocurridos o plantean sus modelos sobre casos hipotéticos futuros y, (iv) el tipo de desastre que estudiaron los autores. La información obtenida se presenta de forma resumida en la tabla 3.

\begin{tabular}{|c|c|c|c|c|}
\hline Autores & $\begin{array}{l}\text { Alcance del } \\
\text { estudio }\end{array}$ & Zona geográfica & Énfasis del estudio & Tipo de desastre \\
\hline Balcik y Beamon (2008) & General & No especifica & Eventos posteriores & Terremoto \\
\hline Chakravarty (2014) & General & No menciona & Eventos posteriores & Huracán \\
\hline Kunz, et al. (2014) & General & No menciona & Casos previos & No especifica \\
\hline Najafi, et al. (2015) & General & No menciona & Eventos posteriores & No especifica \\
\hline Yang y Zhu (2016) & General & No menciona & Eventos posteriores & No especifica \\
\hline Bell, et al. (2014) & Específico & Sichuan, China & Eventos posteriores & Terremoto \\
\hline Edrissi, et al. (2015) & Específico & Teherán, Irán & Eventos posteriores & Terremoto \\
\hline Eftekhar, et al. (2014) & Específico & $\begin{array}{l}\text { Sudán, Afganistán y } \\
\text { Etiopía }\end{array}$ & Casos previos & $\begin{array}{l}\text { Programa de } \\
\text { desarrollo } \\
\text { humanitario }\end{array}$ \\
\hline Garrido, et al. (2015) & Específico & Chile & Casos previos & Inundación \\
\hline Hong, et al. (2015) & Específico & Sur de USA & Eventos posteriores & Huracán \\
\hline Renkli y Durán (2015) & Específico & Estambul, Turquía & Eventos posteriores & Terremoto \\
\hline $\begin{array}{l}\text { Rodríguez-Espíndola y } \\
\text { Gaytán (2015) }\end{array}$ & Específico & Villahermosa, México & Casos previos & Inundación \\
\hline Salman y Yücel (2015) & Específico & Estambul, Turquía & Eventos posteriores & Terremoto \\
\hline Tuzkaya, et al. (2015) & Específico & Turquía & Eventos posteriores & $\begin{array}{l}\text { Varios desastres } \\
\text { naturales }\end{array}$ \\
\hline $\begin{array}{l}\text { Vargas-Flórez, et al. } \\
\text { (2015) }\end{array}$ & Específico & Perú & Eventos posteriores & $\begin{array}{l}\text { Varios desastres } \\
\text { naturales }\end{array}$ \\
\hline Verma y Gaukler (2015) & Específico & California, USA & Casos previos & Terremoto \\
\hline Zhen, et al. (2015) & Específico & Shanghái, China & Casos previos & No especifica \\
\hline
\end{tabular}

Tabla 3. Características de los estudios enfocados a las etapas de mitigación y preparación. 
Como puede verse en la tabla 3, la mayoría de los estudios abordan casos concretos, mientras que solo 4 autores proponen modelos de carácter general. La figura 3 presenta la distribución por regiones geográficas donde se aplicaron los modelos propuestos en los casos específicos. Puede verse que la mayoría de los estudios están concentrados en los continentes de Asia y Europa, y otra buena parte de casos están en Norteamérica y África, mientras que solo 2 casos, que corresponden al $10 \%$ de los estudios revisados, aplican sus modelos en Suramérica, concretamente en los países de Chile y Perú.

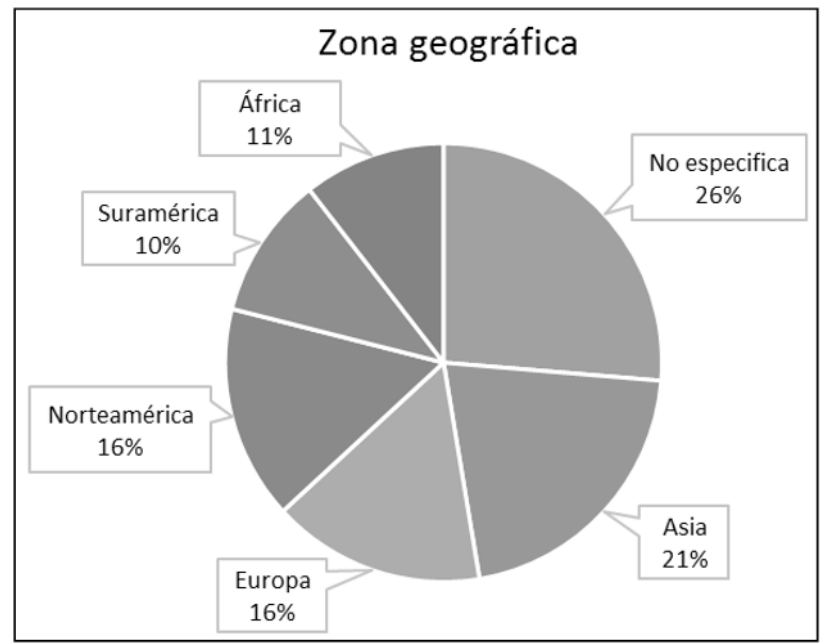

Figura 3. Distribución por zona geográfica de los modelos propuestos en las etapas de mitigación y prevención.

En lo que respecta a la intencionalidad de la contribución, en un 35,2\% de los artículos revisados los autores buscan validar sus modelos con información de eventos pasados, mientras que 11 trabajos (el $64,8 \%$ de los artículos), proponen modelos de preparación para la atención de desastres futuros.

Con relación al tipo de desastre tratado, puede verse que 4 modelos, el $23 \%$ de los autores, no especifican ningún tipo de evento en sus propuestas, mientras que 2 contribuciones presentan diseños para cualquier tipo de desastre natural. Como puede observarse en la figura 4, los terremotos son los desastres más estudiados, debido principalmente a que las regiones definidas para los modelos, son propensas a este tipo de fenómenos (Bell, et al., 2014; Salman \& Yücel, 2015; Renkli \& Durán, 2015), además por el carácter aleatorio que presentan estos eventos y por el nivel de afectación que tiene sobre las vías de transporte (Bell, et al., 2014; Edrissi, et al., 2015). Otros desastres estudiados en la literatura son los huracanes e inundaciones, mientras que sólo una publicación se enfocó en un programa de desarrollo humanitario de largo plazo.

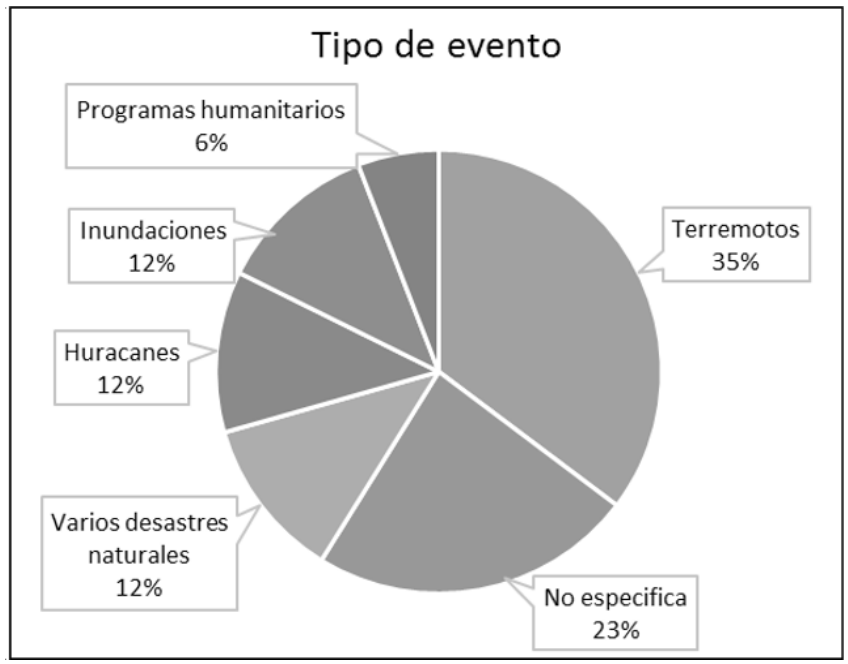

Figura 4. Tipos de eventos tratados en los modelos de preparación y mitigación de desastres.

\section{Conclusiones}

La revisión de literatura desarrollada permite definir que las etapas previas a la ocurrencia y respuesta ante un desastre tienen gran importancia dentro del proceso de la logística humanitaria, pues permiten tomar anticipadamente las decisiones logísticas, desarrollar programas de prevención y adelantar planes de acción de respuesta, creando así las condiciones para que se logren los objetivos de eficiencia y eficacia en la respuesta al desastre, contribuyendo a la minimización del sufrimiento de las víctimas. Sin embargo, otro resultado de la revisión muestra que la mayoría de los autores se centra en las fases posteriores al desastre, mien- 
tras que las etapas de mitigación y preparación son las menos abordadas en la literatura relacionada con el tema (solo el 35\% de la base de artículos consultada).

Con base en el enfoque jerárquico de la planeación de las operaciones, que es común a los sistemas logísticos y de producción, se concluye que las etapas de mitigación y preparación equivalen al nivel estratégico de la gestión, pues se ocupan del diseño del sistema necesario para dar respuesta a las víctimas del desastre, a partir de decisiones como identificación y capacitación de actores, localización de centros, diseño de rutas, políticas para la gestión de inventarios, entre otras.

Se comprueba la preponderancia de los estudios con enfoque cuantitativo, ya que el $61 \%$ de las publicaciones desarrollan modelos matemáticos para la toma de decisiones, centrados en su mayoría en decisiones de pre-localización de centros humanitarios, la gestión de inventarios y definición de rutas de evacuación y distribución durante la etapa de respuesta al desastre.

Aunque se proponen modelos para eventos futuros, son pocos los autores que diseñan modelos de aplicación general, lo cual se explica por la necesidad de disminuir los niveles de complejidad e incertidumbre, por lo que la mayoría de los artículos revisados se refiere a un tipo de desastre específico. De estos, la mayoría de estudios se concentra en países de Asia, Europa y Norteamérica, mientras que los desastres naturales más documentados son los terremotos, seguidos por huracanes e inundaciones.

Como líneas de investigación futura, se propone ampliar la investigación relacionada con las etapas de mitigación y preparación, haciendo énfasis en otros tipos de desastres naturales, cuya participación es mínima en las contribuciones estudiadas. Entre estos eventos, pueden ser tratados los fenómenos relacionados con la variabilidad climática y también los desastres causados por el hombre. Así mismo, es importante contribuir a la comprensión y manejo de desastres en otros contextos geográficos, especialmente en países latinoamericanos, cuya participación en la literatura revisada resulta muy escasa. Estos elementos constituyen un fuerte campo de interés para los investigadores hacia futuros estudios.

\section{Referencias}

Abidi, H., de Leeuw, S., \& Klumpp, M. (2014). Humanitarian Supply Chain Performance Management: A Systematic Literature Review. Supply Chain Management: An International Journal, 19 (5/6), 592608. doi: 10.1108/SCM-09-2013-0349

Afsar, H. M., Prins, C., \& Santos, A. C. (2014). Exact and heuristic algorithms for solving the generalized vehicle routing problem with flexible fleet size. International Transactions in Operational Research, 21 (1), 153-175. doi: 10.1111/itor.12041

Altay, N., \& Green, W. G. (2006). OR/MS research in disaster operations management. European Journal of Operational Research, 175 (1), 475-493. doi: 10.1016/j.ejor.2005.05.016

Anaya-Arenas, A. M., Renaud, J., \& Ruiz, A. (2014). Relief distribution networks: a systematic review. Annals of Operations Research, 223 (1), 53-79. doi: 10.1007/s10479-014-1581-y

Balcik, B. \& Beamon, B. M. (2008). Facility location in humanitarian relief. International Journal of Logistics: Research and Applications, 11 (2), 101-121. doi: 10.1080/13675560701561789

Baldini, G., Oliveri, F., Braun, M., Seuschek, H., \& Hess, E. (2012). Securing disaster supply chains with cryptography enhanced RFID. Disaster Prevention and Management: An International Journal, 21 (1), 51-70. doi: 10.1108/09653561211202700

Barbarosoğlu, G., \& Arda, Y. (2004). A two-stage stochastic programming framework for transportation planning in disaster response. Journal of 
the Operational Research Society, 55 (1), 43-53. doi: 10.1057/palgrave.jors.2601652

Bell, M. G. H., Fonzone, A., \& Polyzoni, C. (2014). Depot location in degradable transport networks. Transportation Research Part B-Methodological, 66, 148-161. doi: 10.1016/j.trb.2013.11.003

Camacho-Vallejo, J. F., González-Rodríguez, E., Almaguer, F. J., \& González-Ramírez, R. G. (2015). A bi-level optimization model for aid distribution after the occurrence of a disaster. Journal of Cleaner Production, 105, 134-145. doi: 10.1016/j.jclepro.2014.09.069

Chakravarty, A. K. (2014). Humanitarian relief chain: Rapid response under uncertainty. International Journal of Production Economics, 151 (1), 146-157. doi: 10.1016/j.ijpe.2013.10.007

Das, R., \& Hanaoka, S. (2014). Relief inventory modelling with stochastic lead-time and demand. European Journal of Operational Research, 235 (3), 616-623. doi: 10.1016/j.ejor.2013.12.042

Day, J. M., Melnyk, S. A., Larson, P. D., Davis, E. W., \& Whybark, D. C. (2012). Humanitarian and disaster relief supply chains: a matter of life and death. Journal of Supply Chain Management, 48 (2), 21-36. doi: $10.1111 / j .1745-493 X .2012 .03267 . x$

Edrissi, A., Nourinejad, M., \& Roorda, M. J. (2015). Transportation network reliability in emergency response. Transportation Research Part E-Logistics and Transportation Review, 80, 56-73. doi: 10.1016/j.tre.2015.05.005

Eftekhar, M., Masini, A., Robotis, A., \& Van Wassenhove, L. N. (2014). Vehicle Procurement Policy for Humanitarian Development Programs. Production and Operations Management, 23 (6), 951-964. doi: 10.1111/poms. 12108

FEMA. (2010). The Four Phases Of Emergency Management. Recuperado de: https://training.fema. gov/emiweb/downloads/is10_unit3.doc
Garrido, R. A., Lamas, P., \& Pino, F. J. (2015). A stochastic programming approach for floods emergency logistics. Transportation Research Part E-Logistics and Transportation Review, 75, 18-31. doi: 10.1016/j.tre.2014.12.002

Gutjahr, W. J., \& Dzubur, N. (2016). Bi-objective bilevel optimization of distribution center locations considering user equilibria. Transportation Research Part E-Logistics and Transportation Review, 85, 1-22. doi: 10.1016/j.tre.2015.11.001

Hadiguna, R. A., Kamil, I., Delati, A., \& Reed, R. (2014). Implementing a web-based decision support system for disaster logistics: A case study of an evacuation location assessment for Indonesia. International Journal of Disaster Risk Reduction, 9, 38-47. doi: 10.1016/j.ijdrr.2014.02.004

Haghani, A., \& Oh, S. C. (1996). Formulation and Solution of a Multi-Commodity, Multi-Modal Network Flow Model for Disaster Relief Operations. Transportation Research Part A-Policy and Practice, 30 (3), 231-250. doi: 10.1016/0965-8564(95)000208

Holguín-Veras, J., Pérez, N., Jaller, M., Van Wassenhove, L. N., \& Aros-Vera, F. (2013). On the appropriate objective function for post-disaster humanitarian logistics models. Journal of Operations Management, 31 (5), 262-280. doi: 10.1016/j. jom.2013.06.002

Hong, X., Lejeune, M. A., \& Noyan, N. (2015). Stochastic Network Design for Disaster Preparedness. IIE Transactions, 47 (4), 329-357. doi: 10.1080/0740817X.2014.919044

Hoyos, M. C., Morales, R. S., \& Akhavan-Tabatabaei, R. (2015). OR models with stochastic components in disaster operations management: A literature survey. Computers \& Industrial Engineering, 82, 183197. doi: 10.1016/j.cie.2014.11.025

İvgin, M. (2013). The decision-making models for relief asset management and interaction with disaster mitigation. International Journal of Di- 
saster Risk Reduction, 5, 107-116. doi: 10.1016/j.ijdrr.2013.08.005

Kovács, G., \&Spens, K.M. (2007). Humanitarian logistics in disaster relief operations. International Journal of Physical Distribution \& Logistics Management, 37 (2), 99-114. doi: 10.1108/09600030710734820

Kovács, G., \& Spens, K. M. (2009). Identifying challenges in humanitarian logistics. International Journal of Physical Distribution \& Logistics Management, 39 (6), 506-528. doi: 10.1108/09600030910985848

Kunz, N., Reiner, G., \& Gold, S. (2014). Investing in disaster management capabilities versus pre-positioning inventory: A new approach to disaster preparedness, International Journal of Production Economics, 157, 261-272. doi: 10.1016/j. ijpe.2013.11.002

Lassiter, K., Khademi, A., \& Taaffe, K. M. (2015). A robust optimization approach to volunteer management in humanitarian crises. International Journal of Production Economics, 163, 97-111. doi: 10.1016/j.ijpe.2015.02.018

Liu, Y., \& Guo, B. (2014). A Lexicographic Approach to Postdisaster Relief Logistics Planning Considering Fill Rates and Costs under Uncertainty. Mathematical Problems in Engineering. Article ID 939853, 17. doi: $10.1155 / 2014 / 939853$

Matopoulos, A., Kovács, G., \& Hayes, O. (2014). Local Resources and Procurement Practices in Humanitarian Supply Chains: An Empirical Examination of Large-Scale House Reconstruction Projects. Decision Sciences, 45 (4), 621-646. doi: 10.1111/ deci.12086

Maya-Duque, P. A., Dolinskaya, I. S., \& Sörensen, K. (2016). Network repair crew scheduling and routing for emergency relief distribution problem. European Journal of Operational Research, 248 (1), 272-285. doi: 10.1016/j.ejor.2015.06.026
Najafi, M., Farahani, R. Z., De Brito, M. P., \& Dullaert, W. (2015). Location and Distribution Management of Relief Centers: A Genetic Algorithm Approach. International Journal of Information Technology \& Decision Making, 14 (4), 769-803. doi: 10.1142/ S0219622014500382

Oloruntoba, R., \& Gray, R. (2009). Customer service in emergency relief chains. International Journal of Physical Distribution \& Logistics Management, 39 (6), 486-505. doi: 10.1108/09600030910985839

Özdamar, L., \&Ertem, M. A. (2015). Models, solutions and enabling technologies in humanitarian logistics. European Journal of Operational Research, 244 (1), 55-65. doi: 10.1016/j.ejor.2014.11.030

Ozguven, E. E., \& Ozbay, K. (2015). An RFID-based inventory management framework for emergency relief operations. Transportation Research Part C-Emerging Technologies, 57, 166-187. doi: 10.1016/j.trc.2015.06.021

Pazirandeh, A. (2011). Sourcing in global health supply chains for developing countries. International Journal of Physical Distribution \& Logistics Management, 41 (4), 364-384. doi: 10.1108/09600031111131931

Pettit, S., \& Beresford, A. (2009). Critical success factors in the context of humanitarian aid supply chains. International Journal of Physical Distribution \& Logistics Management, 39 (6), 450-468. doi: $10.1108 / 09600030910985811$

Rancourt, M. Ė., Cordeau, J. F., Laporte, G., \& Watkins, B. (2015). Tactical network planning for food aid distribution in Kenya. Computers \& Operations Research, 56, 68-83. doi: 10.1016/j.cor.2014.10.018

Renkli, Ç., \& Duran, S. (2015). Pre-Positioning Disaster Response Facilities and Relief Items. Human and Ecological Risk Assessment: An International Journal, 21 (5), 1169-1185. doi: $10.1080 / 10807039.2014 .957940$ 
Rivera, J. C., Afsar, H. M., \& Prins, C. (2015). A multistart iterated local search for the multitrip cumulative capacitated vehicle routing problem. Computational Optimization and Applications, 61 (1), 159-187. doi: 10.1007/s10589-014-9713-5

Robledo, S., Osorio, G. A., \& López, C. (2014). Networking en pequeña empresa: una revisión bibliográfica utilizando la teoría de grafos. Revista Vínculos, 11 (2), 6-16.

Rodríguez-Espíndola, O., \& Gaytán, J. (2015). Scenario-based preparedness plan for floods. Natural Hazards, 76 (2), 1241-1262. doi: 10.1007/s11069014-1544-2

Rottkemper, B., Fischer, K., Blecken, A., \& Danne, C. (2011). Inventory relocation for overlapping disaster settings in humanitarian operations. $O R$ Spectrum, 33 (3), 721-749. doi: 10.1007/s00291011-0260-5

Salman, F. S., \& Yücel, E. (2015). Emergency facility location under random network damage: Insights from the Istanbul case. Computers \& Operations Research, 62, 266-281. doi: 10.1016/j.cor.2014.07.015

Scholten, K., Scott, P. S., \& Fynes, B. (2014). Mitigation processes - antecedents for building supply chain resilience. Supply Chain Management: An International Journal, 19 (2), 211-228. doi: 10.1108/ SCM-06-2013-0191

Sharif, M. T., \& Salari, M. (2015). A GRASP algorithm for a humanitarian relief transportation problem. Engineering Applications of Artificial Intelligence, 41, 259-269. doi: 10.1016/j.engappai.2015.02.013

Tuzkaya, U. R., Yilmazer, K. B., \& Tuzkaya, G. (2015). An Integrated Methodology for the Emergency Logistics Centers Location Selection Problem and its Application for the Turkey Case. Homeland Security \& Emergency Management, 12 (1), 121-144. doi: 10.1515/jhsem-2013-0107
Tzeng, G. H., Cheng, H. J., \& Huang, T. D. (2007). Multi-objective optimal planning for designing relief delivery systems. Transportation Research Part E-Logistics and Transportation Review, 43 (6), 673686. doi: 10.1016/j.tre.2006.10.012

Ülkü, M. A., Bell, K. M., \& Wilson, S. G. (2015). Modeling the impact of donor behavior on humanitarian aid operations. Annals of Operations Research, 230 (1), 153-168. doi: 10.1007/s10479014-1623-5

Van Wassenhove, L. N. (2006). Humanitarian aid logistics: supply chain management in high gear. Journal of the Operational Research Society, 57 (5), 475-489. doi: 10.1057/palgrave.jors.2602125

Vargas-Florez, J., Lauras, M., Okongwu, U., \& Dupont, L. (2015). A decision support system for robust humanitarian facility location. Engineering Applications of Artificial Intelligence, 46 Part B, 326335. doi: 10.1016/j.engappai.2015.06.020

Verma, A., \& Gaukler, G. M. (2015). Pre-positioning disaster response facilities at safe locations: An evaluation of deterministic and stochastic modeling approaches. Computers \& Operations Research, 62, 197-209. doi: 10.1016/j.cor.2014.10.006

Yang, X., \& Zhu, S. (2016). Solution to the Multidepot Inventory Slack-Routing Problem at the Planning Stage. Journal of Computing in Civil Engineering, 30 (1). doi: 10.1061/(ASCE)CP.19435487.0000449

Yi, W., \& Özdamar, L. (2007). A dynamic logistics coordination model for evacuation and support in disaster response activities. European Journal of Operational Research, 179 (3), 1177-1193. doi: 10.1016/j.ejor.2005.03.077

Zhen, L., Wang, K., \& Liu, H. C. (2015). Disaster Relief Facility Network Design in Metropolises. IEEE Transactions on Systems, Man, and Cybernetics: Systems, 45 (5), 751-761. doi: 10.1109/TSMC.2014.2364550 